Molybdenum cofactor deficiency, with resultant sulfite oxidase and xanthine dehydrogenase deficiencies, is an inherited, autosomal recessive, inborn error of metabolism manifesting with neonatal seizures, opisthotonus, craniofacial dysmorphic features, ectopia lentis, and progressive, usually fatal, neurologic deterioration. Although rare, with a total of approximately 15 cases reported, the diagnosis is probably overlooked or delayed, as in the present casereport. Additional reports, not cited by the author, include an infant showing moderate short-term clinical improvement with a diet low in methionine and supplemented with cysteine. (see Progress in Pediatric Neurology Vol II, PNB Publ, 1994;pp474-476; Ped Neur Briefs August 1998;12:58-59). In the US, sulfite dipsticks (Quantofix, Maehery-Nagel) may be purchased at $\$ 20 / 100$ through Gallard-Schlesinger, Tel:516-333-5600.

\title{
STARING SPELLS: EPILEPTIC OR NONEPILEPTIC EVENTS
}

A parental questionnaire containing 25 items to distinguish epileptic (absence seizures [AS]) and nonepileptic staring [NES] characteristics in 40 children presenting with staring spells was tested at the Cleveland Clinic, Ohio. Results from 17 children with AS and 23 with NES, diagnosed by neurologic exam, EEG, and video EEG, were compared. Features with high specificity for NES (responsive to touch, uninterrupted play, event recognized by teacher or health professional) occurred more frequently in NES than in AS. Each of these features had moderate sensitivity. Body rocking occurred only in patients with NES, but sensitivity was low. Features with high specificity for AS included limb twitches, upward gaze, and urinary incontinence during staring episode; but sensitivity for these features was low. Problems with learning or attention were reported in $40 \%$ of children in both groups. A more frequent positive family history for staring or AS in the AS group was not significant. (Rosenow F, Wyllie E, Kotagal P et al. Staring spells in children: descriptive features distinguishing epileptic and nonepileptic events. I Pediatr Nov 1998;133:660-663). (Reprints: Elaine Wyllie MD, Department of Neurology, S51, Cleveland Clinic Foundation, 9500 Euclid Ave, Cleveland, OH 44195).

COMMENT. A history of staring spells is often elicited during the pediatric neurology evaluation of children presenting with short attention, distractibility, and other symptoms of ADHD. The need for an EEG in patients with ADHD and in those presenting with staring spells requires clinical judgement. The questionnaire employed in the above report assists in the differentiation of nonepileptic staring (NES) events and absence epilepsy, and offers a group of features that may be considered strongly exclusive of a diagnosis of epilepsy. The diagnosis of NES is most likely in children who respond to touch while staring, continue playing, exhibit body rocking, and are initially identified by a teacher, nurse, or psychologist. In a child with these clinical features and a normal interictal EEG, a video recording may be deferred. The occurrence of associated limb twitching, upward eye movements, interrupted play, or urinary incontinence are indicative of absence epilepsy, and EEG documentation of epileptiform discharges, with or without video-recording, is essential.

Epileptic seizures temporally associated with nonepileptic seizures are reported in 4 patients, one child and 3 adults, evaluated at the New York University Medical Center (Devinsky O, Gordon E. Neurology Nov 1998;51:1293-1296). A 12-year-old girl with medically refractory partial seizures since age 8 years had video-EEG documented epileptic and nonepileptic 
conversion seizures. Attacks were characterized by auditory hallucinations, vertigo, automatisms, screaming, and impaired consciousness. A simple partial seizure progressed to a complex partial seizure and then evolved into a NES. She had a history of sexual abuse. MRI revealed right frontoparietal dysplasia, an area critical for impulse control and behavioral inhibition. The epileptic seizure may trigger the conversion NES, especially in a patient with right frontal lobe pathology.

\section{EMERGENCY CT IN NEW ONSET SEIZURES}

A retrospective analysis of 107 neurologically normal children with emergency brain scans for new-onset seizures is reported from the Children's National Medical Center, Washington, DC. In $8(7 \%)$, the seizures were nonepileptic (gastroesophageal reflux, syncope, rigor). In 49 of the remaining 99 , seizures were provoked (complicated febrile seizure, encephalitis, toxic or metabolic), and in 50, they were unprovoked. CT abnormalities identified in 19 required further investigation or intervention in 7 (tumors in 2, vascular anomalies in 3 , cysticercosis in 1 , and obstructive hydrocephalus in 1). First seizures unprovoked by fever or metabolic causes, and especially focal seizures, were most likely to have underlying pathologies demonstrated on emergency CT and requiring intervention. (Garvey MA, Gaillard WD, Rusin JA et al. Emergency brain computed tomography in children with seizures: who is most likely to benefit? I Pediatr Nov 1998;133:664-669). (Reprints: WD Gaillard MD, Department of Neurology, Children's National Medical Center, 111 Michigan Ave, NW, Washington, DC 20010).

COMMENT. Emergency CT is indicated in a previously well child with a newonset unprovoked seizure, especially if the seizure is focal. A first seizure with fever is unlikely to be symptomatic of an unexpected brain lesion demonstrable by CT and requiring intervention. A careful neurologic exam performed within 24 hours of the seizure, to exclude localized abnormalities and asymmetry of signs, is essential in determining the need for CT. When the history is unreliable, the neurologic signs of uncertain significance, and follow-up unavailable, a CT is advisable before the patient is discharged.

\section{EPILEPSY SURGERY OUTCOME}

Seizure outcome was studied in 136 children receiving surgery for intractable epilepsy at the Cleveland Clinic between 1990 and 1996. The postoperative follow-up period was 1 to 7.5 years (mean, 3.6 years). Cortical dysplasia and low-grade tumor were the most common causes; hippocampal sclerosis was rare. A seizure-free outcome achieved in $60-69 \%$ of patients was more frequent in patients requiring temporal resections (74-80\%) than in those with extratemporal or multilobar resection (52-58\%), and also among patients with tumor (82\%) compared to those with cortical dysplasia (52\%). Age was not a factor; the frequency of a seizure-free outcome was similar for infants, children, and adolescents, and comparable to reports of adult series. (Wyllie E, Comair YG, Kotagal $\mathrm{P}$ et al. Seizure outcome after epilepsy surgery in children and adolescents. Ann Neurol Nov 1998;44:740-748). (Respond: Dr Wyllie, Pediatric Epilepsy Program, The Cleveland Clinic Foundation, 9500 Euclid Ave, Cleveland, OH 44195).

COMMENT. The risk/benefit ratio for surgery of intractable epilepsy varies with the pathology and localization of the lesion. Patients with low-grade tumors and foci located in the temporal lobe have a better outcome than those with cortical dysplasia and extratemporal or hemispheric localization. Outcome is not 